\title{
ESTILOS DE APEGO DE MUJERES QUE PERMANECIERON \\ EXPUESTAS A SITUACIONES DE VIOLENCIA EN SUS RELACIONES \\ DE NOVIAZGO DURANTE LA ADOLESCENCIA TARDÍA.
}

\author{
STYLES OF ATTACHMENT OF WOMEN WHO REMAINED EXPOSED \\ TO SITUATIONS OF VIOLENCE IN THEIR COURTSHIP \\ RELATIONSHIPS DURING LATE ADOLESCENCE.
}

\begin{abstract}
Cindy Artavia-Fallas. ${ }^{1}$
Marco Carranza-Morales. ${ }^{2}$
\end{abstract}

Recibido: 09.06.19

Aprobado: 23.11.19

DOI: $10.15517 /$ isucr.v20i42.41844

\section{Resumen}

El presente estudio aborda el tema de la violencia experimentada por mujeres dentro de relaciones de noviazgo, durante la adolescencia tardía, desde la teoría del apego. Se trabajó con cuatro participantes, para analizar características de los vínculos a lo largo del desarrollo, con el fin de caracterizar el estilo de apego presente en mujeres que permanecieron expuestas a situaciones de violencia dentro de sus relaciones de pareja.

Para lograr lo descrito anteriormente, se utilizaron tres instrumentos. Se recurrió, primero que todo, a una entrevista de historia de vida, para conocer factores de la historia personal que han influido en la construcción del estilo de apego. Posteriormente se aplicó la Entrevista de Apego hacia los Pares (Campos, 2005) y el Autoinforme de Estilos de Apego (Bartolomew y Horowitz, 1991), con el fin de evaluar propiamente el estilo de apego predominante en la persona.

Los resultados muestran estilos de apego inseguro; en dos casos de tipo temeroso y en dos, ansioso. Esto se relaciona con la historia personal de cada participante, con factores de género y el entorno sociocultural. Analizar la dinámica de estos elementos permite comprender su influencia en la permanencia en relaciones de violencia, y así lograr un análisis integral del tema.

\footnotetext{
${ }^{1}$ Costarricense, psicóloga clínica, educadora y gerontóloga, docente en la Escuela de Formación Docente de la Universidad de Costa Rica. Email: cindyaf21@gmail.com

${ }^{2}$ Costarricense, psicólogo, docente en la Escuela de Psicología de la Universidad de Costa Rica. Email:

marco.carranzamorales@gmail.com
} 
Palabras clave: Apego romántico; relaciones de pareja; violencia basada en género; mujeres; adolescencia tardía; adultez emergente.

\section{Abstract}

This study addresses the issue of violence experienced by women in their dating relationships during late adolescence from the attachment theory perspective. Four participants were involved in the study with the purpose of analyzing the characteristics of the bonds throughout their stages of development. This way it was possible to characterize attachment styles of women who remained exposed to abusive situations.

To achieve this, it was necessary to work with three instruments. First of all, a life story interview was used, in order to identify personal characteristics that have an important influence on attachment styles. Second, it was used an attachment styles interview called Entrevista de Apego a los Pares (Campos, 2005), and the Attachment Styles Questionnaire (Bartholomew \& Horowitz, 1991).

The results displayed insecure attachment styles; there were two fearful type cases and two anxious ones. This relates to the personal history of each participant, involving gender factors and the sociocultural environment. By analyzing the dynamics of these elements, their influence on staying in abusive relationships can be understood; in that way, an integrated analysis of the issue can be achieved.

Keywords: Romantic attachment; couple relationships; gender-based violence; women; late adolescence; emerging adulthood.

\section{Introducción}

El presente artículo aborda el tema de la violencia experimentada por mujeres en sus relaciones de pareja durante la adolescencia tardía, tomando como base la investigación de Artavia y Carranza (2012). Se desarrolla el tema desde la teoría del apego, la cual analiza los vínculos afectivos que los seres humanos establecen en diversas etapas de la vida (Bowlby, 1982).

La violencia contra las mujeres se da tanto en relaciones de convivencia o matrimonio, como en relaciones de noviazgo desde edades tempranas. En Costa Rica, la Encuesta Nacional de Violencia contra las Mujeres indica que un $58 \%$ ha sufrido al menos un incidente de violencia física o sexual desde los 16 años y, de éstas, un $24 \%$ ha sufrido esta situación cuatro o más veces en su vida (Sagot y Guzmán, 2004).

Es importante atender estas situaciones durante la adolescencia, pues las manifestaciones de violencia generalmente son muy sutiles y difícilmente identificadas como tales. Además, se sabe que la violencia durante el noviazgo puede predecir la existencia de esta durante el matrimonio o convivencia (Browne y Herbert, 1997; Rodríguez, Antuña y Rodríguez, 2001; Muñoz-Rivas, Graña, O’Leary y González, 2006 citados por Rey, 2008).

Por ello, se exploran las construcciones de las mujeres en torno a sus vínculos, las cuales pueden influir en sus relaciones interpersonales y en la permanencia en relaciones de noviazgo 
donde existe violencia. En este sentido, Mikulincer y Shaver (2007) sostienen que las diferencias en los estilos de apego influyen en el tipo de relaciones que se establezcan y en los comportamientos dentro de estas.

Lo anterior, no indica que se realice un abordaje causa-efecto de la situación, como si un estilo de apego determinara o no la permanencia en una relación en la que existe violencia. Por el contrario, integra la construcción del apego con otras variables como lo son la historia de vida, aspectos del entorno social y roles de género tradicionales.

Un estudio con estas características posibilita una mejor comprensión de un fenómeno tan complejo como es la violencia contra las mujeres, incorporando una nueva perspectiva de estudio y abordaje: la teoría del apego. Integrando la teoría del apego con las teorías sociales ya existentes sobre violencia, puede lograrse un análisis que ayude a complementarlas.

Así, surge la pregunta generadora que orientó la investigación: ¿Cuáles son los estilos de apego y características asociadas a éste, que predominan en mujeres que estuvieron expuestas a una situación de violencia en el noviazgo durante la adolescencia tardía?

A partir de dicha interrogante, se establecieron los siguientes objetivos generales: 1. Caracterizar el estilo de apego presente en cuatro mujeres costarricenses que permanecieron durante la adolescencia tardía en relaciones de noviazgo donde fueron expuestas a situaciones de violencia. 2. Determinar mediante una historia de vida, características de las participantes, del estilo de apego y del contexto, que pueden relacionarse con la permanencia en la relación de noviazgo donde existió violencia por parte de la pareja.

Asimismo, se determinaron los siguientes objetivos específicos: 1.1 Identificar, mediante una entrevista y un instrumento de autoinforme, el estilo de apego predominante cuada una de las mujeres que estuvieron expuestas durante la adolescencia tardía en una relación de noviazgo donde se ejerció violencia por parte de la pareja. 1.2 Establecer las características del estilo de apego que predominan en las mujeres que permanecieron en relaciones en las que estuvieron expuestas a algún tipo de violencia. 2.1 Identificar mediante la historia de vida, características de las participantes que se relacionan con su estilo de apego. 2.2 Evaluar mediante la historia de vida de las participantes, situaciones vitales y de contexto, que pueden haber influido en la construcción de su estilo de apego y en su permanencia en la relación de noviazgo en la que existió violencia por parte de la pareja. 


\section{Sustento Teórico}

Adolescencia tardía y adultez emergente

La adolescencia es un periodo de transición conformado por varias etapas, que se presenta entre la niñez y la edad adulta. En esta fase se define la identidad afectiva, psicológica y social de las personas. Las características de este período se encuentran determinadas, por el contexto social y político de cada época (Coleman y Hendry, 2003; González, 2001). Retana (2007) indica que el contexto familiar en que se desenvuelve la persona influye en su manera de relacionarse con el entorno social y grupo de pares. Señala que en Costa Rica se vive esta etapa como un período para establecer relaciones interpersonales y asumir nuevas responsabilidades que llevan al autoconocimiento.

A raíz de los cambios económicos y sociales producto del proceso de globalización, la forma en que se vive la adolescencia, como transición de la niñez a la vida adulta, no es la misma de hace algunas décadas. Arnett (2000) considera que en el presente, el inicio y el final de la adolescencia no se ven determinados tanto por factores biológicos sino, más bien, por elementos sociales que la definen, según su estructura.

En los países industrializados ha surgido una nueva etapa en el desarrollo de las personas, conocida como adultez emergente y comprende las edades entre 18 y 25 años. Este período abarca edades de la adolescencia tardía (19-21 años) y de la adultez temprana (25-30 años) y comparte características de ambos periodos. Las personas que atraviesan esta etapa manifiestan no sentirse adolescentes pero tampoco experimentar el haber alcanzado la adultez como tal (Arnett, 2000; González, 2001).

Según Arnett (2001), conforme se avanza en la adolescencia, las responsabilidades se hacen cada vez mayores, y la persona adquiere más independencia. Al final de esta etapa, la persona vive múltiples cambios que le llevan a tomar decisiones que pueden marcar su vida de un modo importante, por ello, se ha propuesto el concepto de adultez emergente, pues es una etapa que implica la consolidación de los roles y la identidad de la persona.

Se considera que en este periodo se deben tomar decisiones en ámbitos como el del amor, el trabajo, la visión que se tiene del mundo. Ahora, las relaciones de pareja se vuelven más serias de lo que fueron en la adolescencia temprana, la persona comienza a concebir las relaciones ya no como un espacio para la recreación, sino como un espacio para la intimidad y para conocer con quién le gustaría compartir su vida (Arnett, 2000). 
Se reflejan vínculos más estables y sólidos en las relaciones de las personas que atraviesan esta etapa. Esto es importante para el presente estudio y puede indicar que durante este periodo los vínculos de apego se tornan más fuertes y, en especial, los de pareja.

\section{La teoría del apego}

Los estudios de Bowlby (1983) le llevaron a definir el término de apego como "cualquier forma de comportamiento que hace que una persona alcance o conserve proximidad con respecto a otro individuo diferenciado y preferido" (p. 60). Este concepto ayuda a comprender el establecimiento de vínculos, que en un principio son instintivos y su fin último es la adaptación.

Se ha postulado, que a raíz de la relación que tienen los niños y las niñas con las y los cuidadores primarios, se construyen representaciones mentales sobre quién es la figura de apego, dónde encontrarla y cómo debe responder. Estas representaciones influyen en las relaciones que se construirán posteriormente en la vida de las personas (Carrillo et.al, 2004).

Recientemente, Mikulincer y Shaver (2007) postularon que los estilos de apego consisten en formas de relacionarse con los otros, según las representaciones que se construyan a partir de la relación con las y los cuidadores primarios. Estos autores parten de los postulados de Bowlby (1998) y Ainsworth (1967) sobre la existencia de tres estilos de apego a partir de sus observaciones, con base en las respuestas de las niñas y los niños ante la separación de sus figuras de apego primarias. Consideran que la amenaza de perder el objeto de apego produce ansiedad, que lleva a la persona a realizar lo que sea necesario para preservarlo y mantener el vínculo. Las figuras de apego que se seleccionen dependen de las construcciones de mundo y de sí mismo que elabore cada individuo.

Las investigaciones en torno al apego han continuado por diferentes líneas, partiendo de la teoría inicial de Bowlby. Así, Zimmermann, Maier, Winter y Grossmann (2001) establecen que el sistema de apego consiste en un medio para lograr la regulación del afecto, el cual tiene como meta principal el establecimiento de la seguridad psicológica de las personas.

Se ha postulado que las diferencias individuales en la relación con las y los cuidadores primarios que cada persona desarrolle, influirán en la imagen que se tiene de sí mismo y de los demás. De este modo se desarrollan expectativas, necesidades, emociones y comportamientos sociales como resultado de la historia personal de apego de cada persona; a esto se le entiende como estilos de apego. 
Basándose en ello, Bartholomew (1990; citado por Feeny y Noller, 2001), propone cuatro estilos de apego: seguro, evitativo, ansioso y temeroso, los cuales se postulan según representaciones internas, ya sean positivas o negativas, que se hayan construido sobre sí mismo y los otros. Bartholomew y Horowitz (1991) plantearon características para cada uno de estos estilos de apego.

En el estilo de apego seguro existe comodidad con la intimidad y autonomía. Estas personas valoran las relaciones de apego, pero, a su vez, son independientes. En el estilo de apego ansioso se presenta una fuerte preocupación por las relaciones ante las cuales se siente confusión. Estas personas no se sienten dignas del amor de los demás, por lo que presentan baja autoestima, baja confianza en sí mismos y ambivalencia en sus relaciones.

El estilo de apego evitativo, por su parte, se caracteriza por una constante búsqueda de independencia. Hay poca apertura emocional y se evitan contactos cercanos e intimidad, minimizando la importancia de las relaciones de apego en sus vidas. Sienten que pueden ser rechazados por los otros, lo que hace que ellos mismos se muestren rechazantes con los demás. En el estilo de apego temeroso la persona considera que los demás le rechazarán, por lo que busca protegerse de este rechazo. En estas personas se presenta temor a la intimidad y se evitan los contactos sociales, a la vez que necesitan de los demás para tener una valoración positiva de sí (Bartholomew y Horowitz ,1991).

Feeney y Noller (2001) sostienen que entre la infancia tardía y la adolescencia, es cuando las personas comienzan a experimentar un cambio gradual en el objeto de las conductas de apego. Bowlby (1998) afirma que el vínculo que se establece en la infancia con los padres se transforma en la adolescencia, pues otras personas, fuera del núcleo familiar, comienzan a tener igual o mayor importancia. Hazan y Shaver (1988; citado por Feeney y Noller, 2001) postulan que en la adolescencia las relaciones amorosas pueden considerarse relaciones de apego iguales a las descritas por Bowlby en la infancia, ya que constituyen vínculos con dinámicas emocionales complejas, reflejándose en ellas los estilos de apego de la infancia.

Para Penagos et al, (2006), en las primeras relaciones románticas la persona busca seguridad, cooperación, confianza y afecto en la pareja, por lo que puede producirse ansiedad ante una posible separación del objeto de apego. Lafontaine, Marie-France, Lussier, Yvan (2005) afirman que según el estilo de apego, así será el manejo de las emociones dentro de las relaciones interpersonales, en 
especial, las de pareja, en las que, estilos de apego inseguros pueden influir en situaciones de violencia dentro de la relación.

El apego y los vínculos amorosos son construcciones que buscan el bienestar de la persona y pueden presentarse simultáneamente. No obstante, en los casos por estudiar, existe una relación de violencia que no corresponde con el principio de búsqueda de supervivencia y bienestar que fundamenta la teoría del apego. Por ello, es importante relacionar teoría mencionada con otros factores influyentes en la permanencia en relaciones de pareja en las que existe violencia.

Relaciones de pareja en la adolescencia

Para Adelman y Kil (2007), en la adolescencia se producen las primeras relaciones románticas de la vida. Estas permiten satisfacer los deseos de socialización y construcción de la identidad sexual del individuo. Es una etapa en la que involucrarse en relaciones románticas es importante para la construcción de la identidad, en contextos que les permitan dar paso a aspectos como intimidad, cuidado y compañía (Penagos et al., 2006). Las anteriores son características de las relaciones de apego.

Collins et al., (2009) entienden las relaciones románticas como un conjunto de interacciones en las que dos personas se relacionan por mutuo acuerdo. Se caracterizan por muestras intensas de afecto. En la actualidad, sin embargo, el concepto de relaciones de pareja ha cambiado pues, ya no se espera lo mismo de ellas que en el pasado. Beck y Beck-Gernsheim (2001), por su parte, consideran que los cambios en los papeles y vínculos tradicionales hacen que sea más difícil para las personas identificar su papel dentro de las relaciones de pareja. En las ocasiones en que esta situación no se maneja adecuadamente, la incertidumbre que ella genera es depositada dentro de la relación en sí, lo que puede generar conflictos de pareja.

Estos cambios afectan a la sociedad en general así como a la población adolescente. Para Murillo (1996), jóvenes, en este proceso de consolidación de la identidad, se ven inmersos en una época de cambios sociales, llenos de contradicciones, por lo que a veces no tienen claro qué esperar de las relaciones interpersonales y del establecimiento de vínculos, en especial románticos, que son un ensayo para la vida adulta.

Collins et al. (2009), sostienen que en la etapa de adolescencia pueden presentarse relaciones románticas de baja calidad, en las que existen conductas de irritación, ambivalencia y un excesivo comportamiento de control hacia la pareja; estas situaciones pueden llevar a relaciones de violencia 
dentro de las mismas y a relaciones disfuncionales en la edad adulta. La presencia de relaciones de calidad durante la adolescencia, en las cuales exista una buena comunicación y compromiso, probablemente generará relaciones más estables y positivas durante la adultez. También se considera que la calidad de las relaciones de pareja que mantengan las personas y el tipo de vínculos que se establezcan, están estrechamente relacionadas con el estilo de apego de cada individuo.

Dado que en este periodo las relaciones de pareja comienzan, en ocasiones, a ser más estables, es importante prevenir la violencia desde allí, y estudiar factores de la dinámica que pueden influir en que la misma se perpetúe. A raíz de situaciones conflictivas en las relaciones de pareja adolescentes, se identifican formas básicas de violencia, entre las cuales están el abuso físico, el abuso emocional y abuso sexual.

En síntesis, el establecimiento de relaciones de noviazgo en la etapa adolescente es parte normal del desarrollo. Las maneras en que se relacionan las y los adolescentes son diferentes en la actualidad pues las formas de vinculación tradicionales han quedado atrás.

Raíces de la violencia en la sociedad

Para Hidalgo (2009), la violencia proviene de un contexto social que la propicia y es utilizada por las personas para alcanzar sus fines, buscando por este medio mantener la superioridad frente a otros. Cuando se legitima la violencia, ésta llega a considerarse un derecho de los individuos, convirtiéndose en una forma de relación social y mecanismo de control (Murillo, 1996).

La violencia contra las mujeres proviene de las relaciones desiguales de poder que históricamente han sido establecidas según el género (Hidalgo, 2009). Se han identificado diversas manifestaciones de la violencia:

$\checkmark$ Violencia basada en género: Se trata de una violencia dirigida hacia las mujeres con el fin de incrementar o mantener la subordinación con respecto del género masculino.

$\checkmark$ Violencia doméstica: Es una de las manifestaciones de la violencia de género. Se produce en el ámbito privado del hogar, puede darse en relaciones de noviazgo, de matrimonio o de convivencia.

$\checkmark$ Violencia intrafamiliar: Son las situaciones de abuso de poder dentro del ámbito familiar y que ocasionan a las víctimas daños en diversos niveles. Los grupos más vulnerables de este tipo de violencia son las mujeres, los niños y las personas adultas mayores (Corsi, 2003). 
La presente investigación se centra en el concepto de violencia basada en género y doméstica, en cualquiera de sus manifestaciones:

$\checkmark$ Violencia física: Agresión que incluye golpes, empujones, puñetazos, bofetadas y todo tipo de acción que cause un daño físico en la víctima.

$\checkmark$ Violencia psicológica: Forma de agresión en que se pretende denigrar, controlar y bloquear la autonomía de la víctima.

$\checkmark$ Violencia sexual: Sucede cuando la víctima es obligada a someterse a actividades sexuales contra su voluntad y consentimiento.

$\checkmark$ Violencia patrimonial: Acción que implique perjuicio, pérdida, destrucción de objetos personales, derechos o bienes económicos de la víctima (Ortiz y Morales, 1999; Silva, sf; Solano, 2007).

En ocasiones resulta difícil de identificar la violencia en los ámbitos privados debido a su invisibilización y naturalización. Corsi (2003) considera que la violencia tiene consecuencias tanto a nivel individual como social. Quienes han estado expuestas a situaciones de este tipo pueden presentar diversos trastornos, sentimientos de culpa, depresión, miedos, entre otros. Además, a nivel social la violencia también tiene importantes secuelas en los ámbitos escolar, laboral y de salud.

Cada vez hay una mayor consciencia acerca de la prevalencia y el impacto de la violencia en las relaciones de noviazgo. Marquart et al.(2007) afirman que en los Estados Unidos uno de cada seis estudiantes, entre los niveles de $10^{\circ}$ y $12^{\circ}$ de secundaria, han experimentado violencia en sus relaciones de noviazgo, lo cual, según Black et al., (2008) afecta su salud mental. Helweg-Larsen, Harding y Kleinman, (2008) afirman que un 66\% de jóvenes universitarios, también en los Estados Unidos, han experimentado mientras asistían a secundaria al menos un incidente de violencia física o sexual de parte de su pareja. A nivel nacional se estima que del total de casos de violencia contra las mujeres que se reportan, al menos en un $12 \%$ es perpetrada por un novio o ex novio de la mujer (Sagot y Guzmán, 2004).

Murillo (1996) señala que las conductas violentas se manifiestan generalmente de parte del novio a la novia y Tafalla (2001) afirma que la violencia difícilmente es identificada como tal y se 
confunde con comportamientos de amor (control, celos). Por esto muchas personas continúen en relaciones en que existe violencia sin detectarla.

Dado que los patrones violentos durante el noviazgo de adolescentes pueden llevar al establecimiento de relaciones conflictivas en la vida adulta (Marquart et al. 2007), es importante trabajar esta problemática desde sus primeras manifestaciones.

\section{Estrategia Metodológica}

Se parte de un modelo mixto de investigación con predominancia de un enfoque cualitativo, en el que, tal como señalan Hernández, Fernández y Baptista (2007), se integran estrategias cuantitativas y cualitativas. Sandoval (2002), indica que este abordaje logra un análisis del tema, partiendo formas de pensar, sentir y actuar de las personas participantes.

La investigación se desarrolla con un enfoque fenomenológico, ya busca describir la experiencia sin tomar una posición de causa-efecto (Sandoval, 2002). En este caso interesa el análisis, la descripción y la interpretación de los significados encubiertos, evitando abordar el apego como una variable causal del fenómeno de la violencia en las relaciones de noviazgo durante la adolescencia tardía. Por ello, se analiza la situación de violencia a partir de diversos referentes teóricos y contextuales dentro de los cuales se incluye la teoría del apego.

Se trabaja con un diseño transversal, pues los datos fueron recopilados en un momento único por medio de las entrevistas. Además, consiste en un estudio exploratorio dado que se trabajaron variables de las que se sabía poco (Hernández et al., 2010).

Para la selección de las participantes, se hace un muestreo de casos homogéneos (Sandoval, 2002), y se trabaja con personas que cuenten con algún tipo de vivencia en común, en este caso, haber permanecido en una relación de noviazgo en la que se dio violencia. Se contó con la participación de cuatro mujeres entre 21 y 25 años de edad que permanecieron durante la adolescencia tardía (19-21 años) en una relación romántica heterosexual en que existieran formas de violencia hacia ellas en cualquiera de sus manifestaciones, durante un período mayor a un año. No se trabajó con personas que se encontraran viviendo la situación de violencia en el momento de la investigación, puesto que esto podía requerir de un proceso de intervención en crisis que, por las características del estudio, no era posible brindar.

Se contactó a las participantes por medio de instituciones que brindan atención a mujeres que han enfrentado situaciones de violencia de pareja en Costa Rica, como el Instituto Nacional de las Mujeres (INAMU), con su Delegación de la Mujer y Oficinas de la Mujer; además el Centro de 
Atención Psicológica de la Universidad de Costa Rica. En cada institución se solicitaron permisos, con el fin de recibir información de posibles participantes y se colocó un afiche informativo sobre la investigación; para que, en caso de que alguna mujer se sintiera interesada por participar, pudiera hacer el contacto. Para la protección de las participantes se entregó un consentimiento informado donde se indicaban las características del estudio y se resaltó, que la información obtenida sería totalmente confidencial y que se podría abandonar el proceso si éste le afectaba de modo negativo.

Técnicas e instrumentos utilizados

Se utilizó la Historia de Vida, técnica que, por medio de la recolección de relatos, busca elaborar y transmitir una historia personal o colectiva (Santamarina y Marinas, 1999). Se exploraron elementos relacionados con el desarrollo de la participante: niñez, adolescencia, relaciones intrafamiliares y extrafamiliares, relaciones de apego, relaciones de noviazgo, situaciones de violencia de pareja anteriores, roles de género tradicionales aprendidos.

Para conocer el estilo de apego de las participantes se utilizó la Entrevista de Apego hacia los Pares (EAP) (Campos, 2005), instrumento que cuenta con criterios de validez en el ámbito costarricense, evaluados por medio de pruebas piloto realizadas (coeficientes superiores a .75). Las categorías del instrumento son apropiadas para evaluar el apego hacia la pareja en la adolescencia ya que tal como lo indican Feeney y Noller (2001), tanto las relaciones de pareja como las de amistad, se consideran vínculos de apego pues presentan dinámicas emocionales complejas en las que se reflejan los estilos de apego de la infancia.

El instrumento consiste en una entrevista semiestructurada de 37 preguntas abiertas, divididas en cuatro ejes temáticos: contexto y relación con los padres, percepción y cercanía, reacción ante la separación y confianza y reciprocidad. En estas secciones se exploran a su vez nueve dimensiones de las relaciones interpersonales de la participante con sus pares, familiares o pareja: apertura emocional y confianza, resistencia, valoración personal, valoración del otro, confusión versus coherencia, independencia, enojo, reacción ante la separación y ansiedad.

Ambos instrumentos se complementan, pues uno brinda puntajes respecto a un estilo de apego predominante en la persona a partir de su discurso, mientras que el otro ayuda a analizar la construcción de dicho estilo de apego, a lo largo de la historia personal. Como complemento se utilizó el Autoinforme de Estilos de Apego (AEA) (Bartholomew y Horowitz, 1991) para medir el estilo de apego predominante en las participantes, por medio de afirmaciones que describen el modo en que la persona se relaciona con otros. 
Este último instrumento está conformado por dos partes. En la primera se presentan cuatro afirmaciones que describen el modo de relacionarse con los demás. Cada uno de los párrafos representa un prototipo de apego: seguro, evitativo, ansioso y temeroso. La participante debe elegir una de las opciones, según considere que la misma la representa o no.

En la segunda parte del instrumento se presentan las mismas cuatro afirmaciones del inicio, pero en esta ocasión, se debe seleccionar en una escala Likert con un puntaje de 1 a 7, el grado en que la participante se identifica con cada una de dichas afirmaciones.

El AEA ha tenido éxito en múltiples investigaciones y sus escalas demuestran validez en múltiples culturas. A nivel nacional, el instrumento fue adaptado para adolescentes en su versión castellana por Tapia (en preparación), en el marco de un estudio del Instituto de Investigaciones Psicológicas de la Universidad de Costa Rica.

\section{Procedimiento}

Para recolectar la información se acuerdan dos sesiones con cada participante. En la primera se realiza la historia de vida y en la segunda, la EAP de Campos (2005) y la AEA de (Bartholomew y Horowitz 1991). Aplicar los instrumentos en este orden, permite un abordaje de lo general a lo particular. Una vez hecho esto, se analiza la información, procesando cada instrumento de modo independiente, para posteriormente realizar la triangulación.

La historia de vida se analizó por medio de una codificación de la información por temas y categorías (Navarro y Díaz, 1999). Se utilizó un análisis autobiográfico de la misma, en el que, según Bluck y Habermas (2001), se puede contemplar no sólo el pasado de las participantes sino también las representaciones e impacto que tuvo y tiene éste en su vida. De allí que de los muchos acontecimientos que viven las personas, solamente los más significativos e impactantes son recordados con facilidad.

Para el análisis de la EAP de Campos (2005), el manual del instrumento sugiere transcribir las respuestas obtenidas en una hoja de procesamiento, para analizarlas de acuerdo a las siguientes categorías: Apertura emocional y confianza, Resistencia a la intimidad, Valoración personal, Valoración del otro, Independencia, Enojo, Reacción ante la separación, Confusión versus coherencia y Ansiedad.

El AEA de Bartholomew y Horowitz, (1991) cuenta con un método específico diseñado para su análisis y para cada una de sus partes cuenta con puntajes asignados a cada respuesta. Este instrumento se complementa con la EAP con el fin de contar con dos mediciones del estilo de 
apego, que pudiesen ser contrastadas con la historia de vida y así brindarle más consistencia al estudio, que por ser de un carácter cualitativo, se enriquece con el uso de diversas técnicas de recolección de información.

Una vez hecho esto, se procede a realizar la triangulación, que tal como señala Cisterna (2005), consiste en un método para integrar la información recopilada con la información contenida dentro del marco teórico. Esto permite interpretar los datos obtenidos y la construcción de nuevos conocimientos.

Presentación y análisis de resultados

A continuación se exponen los resultados y análisis de cada participante por separado, para luego integrarlos; los nombres utilizados son pseudónimos.

- "Camila"

Los resultados de Camila en la EAP, indican puntuaciones más altas en un estilo de apego ansioso, sin embargo, en el AEA refleja un apego seguro. Esta diferencia sucedió con tres de las cuatro participantes, lo cual posiblemente se relaciona con el tipo de instrumentos y la forma en que se aplica cada uno de ellos, ya que el AEA, por sus características de autoinforme, y el tipo de preguntas que realiza podría aumentar defensas en las participantes, sesgando con ello sus respuestas.

Al considerar el estilo de apego de las participantes, puede afirmarse que este influye en la no concordancia entre los instrumentos. Por ejemplo Camila, al presentar un estilo de apego ansioso en el cual según Bartholomew y Horowitz (1991), existe una imagen negativa de ella misma y positiva de los demás, hará intentos por contestar el instrumento del modo que refleje una imagen más positivos de ella. Gracias a la historia de vida, fue posible determinar cuál de los resultados resultaba más congruente con el relato. En este caso, el relato de Camila se ajusta más a un estilo de apego ansioso que a un estilo de apego seguro.

En su historia, se refleja una relación más cercana con la madre que con el padre. Comenta que su padre cumplió su rol, desde una posición de control sobre ella: "él siempre ha sido muy posesivo, muy sobreprotector, (...) mi papá nunca nos pegó, él era como amargadillo, (...), nos regañaba y era ese respeto, pero él nunca lo agredió a uno”. Camila construyó su ideal de afectividad, desde lo que vivió en su hogar en la relación con su padre. Desde allí fueron percibidas como muestras de afecto todas las conductas de control y sobreprotección, lo cual espera en sus formas de 
vinculación en la actualidad. Siguiendo con lo que menciona Carrillo et.al, (2004), se observa que Camila construyó a partir de esta etapa, representaciones mentales específicas sobre lo que se debe esperar de las figuras de apego.

Camila indica que su madre asumía una posición pasiva, pero siempre abogando por sus hijas: "es atenta, alcahueta, porque como ella dejó de trabajar para cuidarnos a nosotros, ella nos dice que estudiemos y que ella se encarga de las cosas de la casa”. Podría pensarse que la madre representa un modelo a seguir, mientras que la relación con el padre se consolida como un referente para la construcción de los vínculos, en especial los de pareja.

Dado que Camila, era "muy apegada" a su madre durante la infancia, se le dificultó el ingreso a la educación formal e iniciar nuevas relaciones en el ámbito extrafamiliar: "Cuando entré a la escuela (...) una semana después me agarró mamitis, que no quería y me revolcaba (...) no sé si habrá sido por estar en un lugar grande, con otros chiquitos y otra gente”. Algo similar sucedió con su ingreso al colegio, sin embargo, señala que dejó esta resistencia de lado cuando conoció a su mejor amiga. Este es un paso importante para Camila en la construcción de relaciones fuera del contexto familiar, pues en la infancia no menciona vínculos de este tipo, ya que los mismos se direccionaban más al interior de la familia: "Me acostumbré a estar siempre con mi mamá y mis hermanos jugando".

Lo anterior se puede visualizar desde los postulados teóricos de Delgado, et.al, (2011) en cuanto a que en la etapa de la adolescencia, los pares cobran mayor importancia y se va moldeando, poco a poco, el apego adulto que busca principalmente intimidad, cercanía y apoyo, lo cual encontró en su mejor amiga del colegio que comenzó a funcionar como una figura reguladora de afecto y estabilizadora de la ansiedad, ante la separación de sus figuras primarias de apego.

Camila tuvo su primer novio al ingresar a la universidad, relación que según comenta, fue acelerada, característica del estilo de apego ansioso, en el cual partiendo de Bartholomew y Horowitz (1991) se busca mucha proximidad. Citando a Camila: "no sé si habrá sido como que a uno lo deslumbran porque lo tratan bien o porque tal vez no había tenido nunca una relación seria, entonces yo ya me sentía estable”. En esta relación se presentó violencia, pero ella no fue consciente de esto hasta que, con el paso del tiempo comenzó a detectarla: "Se daban ciertas cosillas de celos (...) por un mensaje o así de un compañero se ponía en cosas (...) él me apartó a mí de mi mejor amiga”. 
Al presentarse la situación de violencia en la primera relación de noviazgo, puede asumirse que Camila no tenía referentes acerca de lo que podía esperar de una pareja, esto, además de su apego ansioso, puede haber influido en la permanencia en esta relación. En las primeras relaciones, Grossman y Grossman (2006) consideran que existen fuertes expectativas de encontrar seguridad, cooperación, confianza y afecto en la pareja. Esto coincide con el relato de Camila en cuanto al ideal de cómo tiene que ser la pareja.

Dado que esta pareja era casi diez años mayor que ella, según comenta, le hacía sentir confianza y estabilidad. Él cumplía roles que ella consideraba de cuido y protección, posteriormente se identifican como mecanismos de control: “¿cómo no detecté eso en el momento?, en su momento de enamoramiento, no lo llegué a ver como un tipo de agresión o de celos”. Como indica Murillo (1997), los roles tradicionales de género, hacen que las mujeres acepten comportamientos de control, pues consideran que cuando los hombres aman deben proteger.

Por el estilo de apego ansioso de Camila, el hecho de sentirse protegida por la pareja favorecía su permanencia en la relación, la cual abandona en el momento en que se sintió desprotegida porque él: "no tenía expectativas de vida". Es en este punto cuando identifica la situación de violencia pues se hizo más explícita: “me dijo que me iba a matar, (...) que se iba a tirar de un puente”. En esta dinámica se refleja su forma de vinculación interpersonal.

\section{- María}

Los resultados de María coincidieron en los dos instrumentos que evalúan el apego. Hay predominancia de un estilo de apego temeroso en la participante, lo cual se evidencia en la historia de vida.

En las relaciones intrafamiliares María menciona haber tenido un vínculo distante con su padre, al cual prácticamente no menciona en su relato y únicamente lo hace cuando se le pregunta directamente. Respecto a su madre afirma que han mantenido una buena relación y los únicos roces que han tenido se dieron durante la adolescencia: “Con mi mamá sí me llevo bien, por ser así la menor". La poca información que facilita Maria podría ser indicador de su estilo de apego temeroso pues, evita la intimidad y los contactos sociales (Bartholomew y Horowitz 1991).

Recuerda que en su infancia tenía muchos amigos de su comunidad y en la escuela, se llevaba bien con la mayoría de personas: "Donde nosotros vivíamos habían como cinco familias pegadas, y tenían hijos de edades parecidas entonces todos salíamos ahí a vacilar, fue muy bonito". En este periodo su estilo de apego temeroso no se ve claramente reflejado. 
Cuando ingresó al colegio, se encontraba atravesando una etapa de cambio de vivienda e institución educativa; en esta última no se sentía cómoda y se le dificultó la adaptación: “Era horrible, las muchachas eran todas pleiteras, las personas eran todas raras, y obviamente estaba acostumbrada a otro tipo de ambiente”.

En este período no refiere haber tenido amistades cercanas y considera que su vínculo más estrecho era un funcionario de su colegio. Lo mismo ocurre en la actualidad, pues establece vínculos mayoritariamente con profesores, o personas que tienen alguna autoridad y que le brindan seguridad y protección: "me hice amiga de la profe, entonces luego conocí otras personas parecidas y mejoró todo". Así, comienza a evidenciar la construcción de su estilo de apego temeroso pues evitaba las relaciones cercanas con pares y la apertura emocional, sin embargo, sí buscaba con determinadas personas la regulación emocional característica de esta etapa (Allen y Manning, 2007).

María menciona que tuvo su primer noviazgo en el colegio; al iniciar esta relación se le dificultó la apertura emocional hacia su pareja (característico de su estilo de apego temeroso), no obstante, con el paso del tiempo aumentó la sensación de dependencia a la relación: "Cuando empezamos, duramos como una semana y él me cortó... fue horrible (...) yo en ese entonces era muy callada, muy tímida y tenía una manera de vida como muy tranquila, muy miedo (...) a mí se me hacía difícil poder hablarle entonces a él no le gustó”. Ante esta situación, ella cambió de actitud para agradarle a esta persona. Esto refleja los roles tradicionales que son reforzados socialmente, y a los que ella debía ajustarse para ser una buena novia.

En esta relación se presentó violencia, pero ella no era consciente, hasta que las peleas se hicieron más frecuentes. Las agresiones y el control que señala de parte de su novio: celarla, ser posesivo y criticarla; acentuaban la imagen negativa de ella misma, característica del estilo de apego temeroso. Esto la llevaba a tolerar la situación pues no era consciente de que él estuviese actuando mal: "Yo siento que él lo hacía demasiado sutil (...) era como: ¡uy! ¿Se va a poner una enagua? (...) ya después a la hora de ponérsela, ya lo pensaba uno”.

Atribuye su permanencia en esta relación a su falta de conocimiento sobre el tema de violencia y al no haber tenido novios antes: "no sé cómo pude soportar tanto, supongo que no sabía, no tenía mucha experiencia (...) y no le tomé importancia”. Tal como señalan Adelman y Kil, (2007), muchas veces en las relaciones en que existe violencia no se tiene claridad de que se trata de esta problemática y se pasan por alto. 
Cuando decide terminar la relación, se le hizo difícil romper el contacto con esta persona: "Duramos como dos años, más los años que uno está ahí, como que está pero no está". Esto posiblemente debido a la ansiedad que genera la posible pérdida del vínculo afectivo en este estilo de apego (Bowlby, 1983). El contacto con la ex pareja se mantuvo hasta que logró desvincularse con menos ansiedad y más evitación.

María ha evitado establecer otra relación de pareja, lo que refleja su estilo de apego temeroso y la dificultad para abrirse emocionalmente a una nueva relación: “Después de esa relación, tratar de volver a tener otra (...) es un caso perdido”.

En síntesis, se lee a través del relato que en el caso de María, la apertura emocional para las relaciones con otras personas se ha dificultado, pero una vez que establece un vínculo cercano tiende a sobrevalorarlo. En el caso de la relación de noviazgo que refiere, parece ser que al igual que con otras relaciones evitó la apertura emocional y la vinculación estrecha, pero, una vez dentro de la relación, aumentó la ansiedad, por lo que el temor a perder a la figura de apego incrementó a tal punto de pasar por alto situaciones de violencia.

\section{- Ana}

En el caso de Ana los instrumentos que evalúan el estilo de apego dieron resultados diferentes uno del otro. La EAP refleja un estilo de apego ansioso, sin embargo, en el AEA, presentó un estilo de apego seguro. Lo sucedido en este caso, podría tener relación con el estilo de apego ansioso de Ana, el cual influye en que al enfrentarse a un instrumento de autoinforme, busque dar una imagen positiva de sí. Esto es característico, según Bartholomew y Horowitz, (1991) del estilo de apego ansioso. Al analizar la historia de vida es posible percatarse de que el discurso de Ana concuerda más con un estilo de apego ansioso y, por consiguiente, con el resultado de la EAP.

En su historia de vida, menciona que su padre abandonó temporalmente el hogar cuando era pequeña, por lo que vivió muchos cambios en su vida, entre ellos cambio de vivienda y de escuela, además de enfrentar problemas económicos; esto la hacía sentirse insegura y desprotegida. El padre, que mantenía contacto con ella, se convirtió, según relata, en una figura intermitente. La relación se volvió inestable, provocándole ansiedad, característica de un apego ansioso, en el cual existe preocupación por las relaciones, en las cuales hay confusión (Bartholomew y Horowitz 1991): “(...) Con mi papá luego de mucho batallar y de ir y venir digamos que ahora nos soportamos, nos toleramos y ahi vamos”. 
Señala que su madre tuvo un rol pasivo en la dinámica que se estaba presentando y esto la llenaba de incertidumbre y la hacía dudar de la disponibilidad de esta, de lo cual se queja hasta la fecha: “Uno no sabe cuándo puede contar con ella y cuándo no”. Su construcción de un estilo de apego ansioso, se relaciona con las figuras de apego inconsistentes que menciona. Desde su infancia percibe que las figuras de apego no estarán disponibles, y se teme a la separación estas (Carrillo et.al, 2004).

Probablemente debido a lo anterior menciona que se le dificultaba la inserción en las instituciones educativas a las que asistía; los constantes cambios de escuela, colegio y el bajo rendimiento académico que, debido a esto presentaba, afectaba su autoestima: "La directora le dijo a mi papá, delante mío, que simplemente a mí no me daba el IQ para llevar dos idiomas y eso aún lo recuerdo con rencor".

Señala que al conocer a su mejor amiga, en colegio, se sintió más segura y continuó sin problema el resto de su etapa escolar. Establecer este vínculo emocional, que se mantiene en la actualidad, le dio más seguridad en las relaciones extrafamiliares que establecía, por lo que constituye una figura de apego muy importante.

En su segundo noviazgo se presentó violencia, la cual llegó a nivel físico. Señala que recibía múltiples críticas y controles por parte de su pareja e indica haber sido consciente de la agresión: "Desde la primera vez que me golpeó ya yo sabía (...) o sea yo sí estaba consciente de que no era una buena persona para mí, pero tampoco lo quería dejar”. Esta situación de violencia física no es frecuente en las relaciones de noviazgo, en las cuales, como lo han postulado Ferrer, et.al, (2008) la violencia suele ser de tipo psicológico o verbal.

A pesar de la gravedad de la situación, Ana la dejaba pasar: "Yo pensaba que si no estaba con él entonces iba a estar sola, como nunca tuve la mejor autoestima del mundo". Como mencionan Bartholomew y Horowitz (1990), en el estilo de apego ansioso se presenta una imagen negativa de sí misma, y positiva de los demás; posiblemente es por ello que en el momento en que alguien demuestra un interés por ella, podría haberse aferrado a la relación sin importar si este vínculo es positivo para ella o no: "Sólo uno que está ahí sabe que uno no lo puede dejar porque no sé cómo explicarlo, es como necesidad”. De este modo la relación finalizó en el momento en que su pareja lo decidió así: "Él terminó la relación por celos y a mí me hacía mucha falta”.

A la dificultad para dejar la relación de violencia se le suma el hecho de que ella, desde su infancia, estuvo inmersa en un contexto en el que el conflicto era parte del diario vivir, por lo que 
salirse de una relación conflictiva es difícil, ya que es la dinámica que ha construido en relación con su vinculación hacia los demás. Así lo expresa refiriéndose al momento en que la relación terminó: "Creo que lo que me hacía falta era el conflicto”.

Posteriormente ha tenido otras relaciones en las que el conflicto ha estado presente. Este caso refleja lo expuesto por la teoría, cuando se plantea que las relaciones de noviazgo en que se produce violencia se encuentran estrechamente relacionadas con estilos de apego inseguros (Mikulincer y Shaver, 2007). La dificultad para desligarse de las figuras de apego, a pesar de las agresiones, se relaciona con vivencias previas y sus construcciones con respecto a los vínculos que establece.

\section{- Sofía}

Al aplicar la EAP, Sofia puntuó alto en un estilo de apego temeroso, sin embargo, al aplicar el AEA puntuó con un estilo de apego evitativo. Este cambio puede reflejar su estilo de apego temeroso y el aumento de defensas al enfrentarse a un instrumento de autoinforme, en el cual, al igual que en la entrevista oral, se evidencia dificultad para la apertura emocional. A lo largo de la historia de vida es posible encontrar puntos que reflejan más la tendencia de un estilo de apego temeroso que evitativo.

Al referirse a su infancia Sofía comenta que existieron conflictos familiares. Describe a su padre como una persona responsable pero afectivamente distante: "Mi papá siempre ha sido muy responsable, pero hubo un tiempo tal vez en el que no fue como muy apegado con nosotros". Expresa haber tenido buenas relaciones con la madre pero según comenta, ella cumplía un rol ambivalente en el sistema familiar. Dentro de la relación de pareja, menciona que asumía un papel más pasivo, sin embargo, en la relación con los hijos era más autoritaria. A pesar de ello comenta que en la familia quien tenía la última palabra siempre fue el padre.

Sus representaciones en torno a lo que puede esperar de las figuras de apego, tal como señalan Bartholomew y Horowitz (1991), se encuentran relacionadas con sus vivencias tempranas, en las que entendía como afecto la responsabilidad del padre en cuanto a necesidades básicas más que a la cercanía afectiva, en sí misma. Esto puede verse reflejado en relaciones posteriores a lo largo del ciclo vital.

Sofía manifiesta haber tenido muchas relaciones con pares durante la infancia. Considera esta, la mejor etapa de su vida y sus interacciones iban más allá del núcleo familiar y se extendían al barrio: "Para mí fue lo mejor (...), todos crecimos juntos, todos los carajillos del barrio (...) para mí fue lo más bonito”. 
Señala que no estableció relaciones cercanas con los pares del colegio, pues mantenía fuertes vínculos con sus vecinos, los cuales, conserva hasta la actualidad. Además, en esta época, el colegio constituía un ambiente poco agradable, donde las dificultades académicas le hacían desconfiar de sí misma como persona.

Debido a su estilo de apego, a Sofía se le dificulta la cercanía emocional con otras personal, debido al alto grado de evitación que presenta. Según Batrholomew y Horowitz, (1991) esto se debe a la visión negativa que tiene de sí, que provoca temor al rechazo. Por esta razón Sofía, aunque teme a los vínculos estrechos, simultáneamente necesita de éstos, para fortalecer una valoración positiva de sí a través de los demás.

Comenta que su primer noviazgo formal se dio con un joven que se encontraba casado y tenía hijos, lo que hizo muy difícil la dinámica y llevó a que la relación terminara: "Fue complicada, él tenía hijos de por medio y yo estaba soltera, entonces había mucho pleito con mi mamá. Él, sí claro, me trataba muy bien, y nos llevamos muy bien (...), pero por los problemas familiares, decidimos mejor seguir cada uno por su lado”.

El hecho de que esta relación terminara tuvo que ver más con factores externos que internos a la misma. Parece ser que en este caso importó más para Sofía la aprobación de sus figuras de apego primarias, que su verdadero deseo de continuar o no dentro de la relación: "Quería que mis padres lo aceptaran".

Posteriormente inició una relación con un compañero de trabajo, en la cual se presentó violencia. Afirma que sufría de maltratos, control y mentiras: "Él era muy celoso, si me llamaban por teléfono decía: ¿con quién está hablando?, a mí me respeta, usted está aquí conmigo”. Esto refleja lo señalado por Marquart et.al, (2007), al afirmar que, en las relaciones de noviazgo, muchas veces, los hombres utilizan la violencia como mecanismo de intimidación y control. Esto no siempre es percibido por las mujeres y en otros casos se justifica, como sucedió con Sofía: "Lo veía normal, yo pensaba que iba a cambiar".

Decidió terminar la relación al percatarse que la situación se salía de sus manos, sin embargo, se le hizo difícil romper el vínculo emocional, y mantuvo contacto con la expareja tiempo después, intentando retomar la relación en varias ocasiones. Su estilo de apego temeroso le dificulta vincularse, al igual que desvincularse por la angustia que según Bartholomew y Horowitz (1991) genera la separación de la figura de apego: “Uno se queda callado, para que la persona no lo deje solo". 
Actualmente Sofía indica que no desea tener pareja, y desde que terminó esa relación ha estado soltera. Parece ser que esta decisión refleja su estilo de apego temeroso, y su negativa a involucrarse, de nuevo, afectivamente con alguien.

Tanto en el caso de Sofía como de las demás participantes, hay poca concientización en torno a la violencia de pareja y sus manifestaciones, por lo que, como señala Tafalla (2001), esta no es percibida como tal y se confunde con amor. Además, en los estilos de apego en que la ansiedad ante la separación es alta, es posible que la misma se evite, aunque la relación sea negativa para la persona. En estos casos parece ser que la pérdida de la figura de apego puede generar más angustia que la situación de violencia en sí misma.

Integración del análisis

Los resultados divergentes obtenidos en la EAP y el AEA, fue un aspecto que dificultó la triangulación de la información obtenida. Ante esta situación, se analizó cuál de los dos resultados respondía mejor y con mayor coherencia al discurso de la participante y su historia de vida. Mediante el análisis de la información cualitativa con que se contaba fue posible determinar que los resultados obtenidos por medio de la EAP eran más consistentes con la historia personal de cada participante. Una vez determinado esto, es posible continuar con la reflexión sobre elementos de la construcción y la manifestación de esta variable.

Tanto Camila como Ana puntuaron en un estilo de apego ansioso. Esto se encuentra muy presente a lo largo de su discurso y es posible visualizar aspectos en común entre ambas participantes. Lo mismo sucedió en el caso de María y Sofía, pues ambas presentan el mismo estilo de apego y manifiestan características en común en sus modos de vinculación y construcción de estilo de apego.

Se ha planteado que los estilos apego se construyen a partir de las relaciones con cuidadores primarios, en las cuales se busca seguridad y protección. (Ammantini y Sergi, 2003). En el caso de todas las participantes existió cierta ambivalencia en la relación con los progenitores desde la infancia, lo cual, generalmente, precede a los estilos de apego inseguro que ellas manifiestan.

En el caso de las participantes, coinciden en el hecho de que, a partir de la relación con el padre, se construyó una concepción de afecto estrechamente relacionada con una conducta de responsabilidad parental en cuanto a necesidades básicas y cuido por parte de éste. No se construyó una dinámica que favoreciera la cercanía afectiva en sí y esto parece reflejarse en relaciones de 
pareja posteriores en la vida. Parece ser que las participantes dejaban pasar por alto las conductas de control que manifestaban sus parejas, siempre y cuando se sintieran protegidas.

En todos los casos, parece haber existido una relación más estrecha con la figura materna, sin embargo, le reprochan en ocasiones su rol pasivo dentro del sistema familiar, el cual, además, ellas mismas han llegado a asumir dentro de sus relaciones de pareja.

Dado que el apego puede cambiar en sus formas y objetos a los que se dirige (Yérnoz et.al, 2001), es importante analizar el modo en que esto se refleja en el discurso de las participantes. En el caso de Camila y Ana, quienes presentan un estilo de apego ansioso, se evidencia más claramente la importancia de estas figuras de apego fuera del núcleo familiar, ya que las mismas manifiestan convertirse en reguladoras del afecto en la vida de estas participantes. Ambas hacen referencia a mejores amigas que desde la adolescencia hasta la actualidad son figuras muy importantes para ellas.

En el caso de María y Sofía, participantes con tendencias de apego temeroso, no se logran identificar con claridad vínculos importantes fuera del núcleo familiar durante la niñez y adolescencia y el vínculo más importante al que hacen referencia es el de pareja.

Por otra parte, respecto a los vínculos de pareja, hay un factor en que todas las participantes coinciden, y es en el hecho de que las cuatro formalizaron una relación de noviazgo hasta etapas avanzadas de la adolescencia. Esto posiblemente influyó en que las relaciones fueran más sólidas en cuanto a compromiso y de más difícil disolución. Estas últimas son, según Arnett, (2001), características de las relaciones de pareja durante la adolescencia tardía y adultez emergente en que se encontraban las participantes. Estas relaciones de pareja, pueden considerarse relaciones de apego, con dinámicas complejas que reflejan los estilos de apego construidos desde la infancia (Feeney y Noller, 2001).

Dado que los relatos de las participantes reflejan el proceso de construcción de los estilos de apego puntuados en la EAP, el estudio se fortalece, demostrando consistencia. Las características de sus estilos de apego se ven reflejadas en su forma de vinculación con otros significativos a lo largo de su ciclo vital.

Las figuras de apego tienen una importante carga simbólica para las personas, por lo que en el caso de estas participantes es fundamental considerar no solamente el vínculo como tal, sino que también las representaciones que se tengan en torno a éste. En los casos analizados se refleja la gran importancia que tenían las parejas de las participantes como figuras mediadoras en la 
regulación del afecto. En estas situaciones se evidencia cómo se depositan muchas expectativas en la relación y al tener tanta carga emocional se dificulta su disolución.

Dado que las cuatro participantes presentan un estilo de apego inseguro, en sus diversas manifestaciones, puede suponerse que esto influye en la permanencia en relaciones en que existe violencia. En este estilo de apego, las personas tienen representaciones mentales negativas de sí mismas y resulta más probable que toleren situaciones en las que su integridad se ve afectada y eviten la ansiedad ante la separación.

Se asume entonces, el apego como un factor que influye en la permanencia en relaciones de pareja en que existe violencia, sin embargo es a su vez, un factor que facilita la salida de este tipo de vinculación. Por ello, resulta importante analizar el contexto y estructura social que influyeron en que esta situación se mantuviera, los cuales se relacionan a su vez con roles tradicionales que desempeñan las personas, según sean mujeres u hombres.

Tal como señala Murillo (1997), las mujeres aceptan los comportamientos de control pues consideran que cuando los hombres aman deben proteger. Además, Castro (2006) afirma que las mujeres tienden a presentar, en su mayoría, estilos de apego ansioso, mientras que los hombres se inclinan más hacia estilos de apego evitativo, lo que se relaciona con factores sociales y culturales. Existe un tipo de interacción diferente que se tiene con hombres y mujeres desde la infancia, tomando el género como punto de partida para la socialización.

En el caso de las participantes, al involucrarse y permanecer en relaciones de noviazgo en las cuales se dio violencia, podrían estar reproduciendo los mandatos sociales y familiares a los cuales han sido expuestas, directa o indirectamente y que han influido en la construcción de su estilo de apego. Además, en su relato se refleja que tenían pocos referentes con respecto a lo que debía ser una relación de pareja, por lo que tendían a idealizarlas, y a ver naturales comportamientos sutiles o evidentes de agresión hacia ellas

De este modo, se postula que, según los datos analizaos, en el caso de las participantes en esta investigación existe una identificación con la figura materna en cuanto a lo que se debe ser y como se debe actuar según su género. Por su parte, el padre influyó como referente de las relaciones y vínculos por establecer con figuras masculinas.

A partir de este análisis se comprende el apego como una construcción no solamente individual sino social, pues se ve influenciado por factores de género, familiares, culturales y contextuales. A 
partir de la dinámica de estas variables se establecen las representaciones que tienen las personas en torno a sí mismos y a los demás, y lo que se puede esperar de sus interacciones.

En síntesis, el hecho de que las participantes permanecieran en relaciones de violencia en las que se encontraban expuestas a situaciones de violencia se relaciona con su estilo de apego, sin desligar éste del contexto social. La conjugación de estos elementos favorece un acercamiento novedoso al tema de violencia partiendo de las características de los vínculos que establecen las personas. Además, se logra establecer un análisis del modo en que interactúan diversos factores dentro de una dinámica en que la mujer ha estado expuesta a una situación de violencia durante el noviazgo.

\section{Reflexiones finales}

Analizar la problemática de la violencia contra las mujeres en el noviazgo desde la teoría del apego permite visualizar aspectos de esta dinámica no solamente partiendo del contexto social que la perpetua, sino desde las construcciones personales que hacen las mujeres como producto de su inserción en dicho contexto, y que influyen en el establecimiento de ciertas formas de vinculación que les pueden dificultar la toma de conciencia y el rechazo de este tipo de relaciones que son conflictivas.

Se debe tener cuidado al plantear una dinámica entre el estilo de apego y la permanencia de mujeres en una relación de violencia pues puede malinterpretarse si no se aclara, desde el inicio, que el estilo de apego y la permanencia en relaciones de noviazgo donde existió violencia no están siendo estudiados de un modo causal pues se están tomando en cuenta múltiples variables. En este caso, fue posible, gracias a la historia de vida, por medio de la cual se logró analizar el contexto sociocultural, el sistema patriarcal y los roles tradicionales de género que se reflejan en el discurso de las participantes y en su apego.

Desde esta posición, se evita el error de creer que un estilo de apego determina la permanencia de las mujeres en una relación en que han sido víctimas de violencia. Por otra parte, se evita justificar a los hombres agresores a partir de la creencia de que es más común un estilo de apego en esta población. Así, no se psicologiza un problema como la violencia de género que tiene orígenes e implicaciones sociales que no pueden ser ignoradas.

$\mathrm{Al}$ analizar el tema de apego de modo integral, se comprenden mejor los postulados de Bowlby (1983), cuando indica que los vínculos con las y los cuidadores primarios se mantienen y se expresan en otras relaciones ya sea con pares o parejas, a lo largo de la vida. En este caso, en el 
discurso de las cuatro participantes se evidencia ambivalencia en el vínculo con el padre a causa de su presencia-ausencia. Esto influye en la construcción de sus tendencias de apego inseguro donde, según Bartholomew y Horowitz (1991), se considera que las figuras de apego no estarán disponibles y se teme a la separación de estas. Parece ser entonces que la figura paterna influye en las representaciones mentales en torno a lo que se espera recibir del otro como pareja. La madre, por su parte, funciona como una figura con quien identificarse en su forma de vinculación con otros.

El hecho de que algunas participantes en este estudio hayan vivido su infancia vinculada casi exclusivamente al contexto intrafamiliar, con pocas relaciones fuera de este ámbito (por ejemplo con pares), parece relacionarse con la construcción de un estilo de apego ansioso en etapas posteriores de la vida. Haber tenido poco contacto extrafamiliar en etapas tempranas del desarrollo puede ser un factor que genere representaciones en torno a sí mismas y a los demás que produzcan ansiedad para posteriores relaciones.

Dado que en el caso de las cuatro participantes, la situación de violencia se presentó en sus primeros noviazgos, podría pensarse que la permanencia en ésta se relaciona con la falta de referentes en torno a lo que es una relación de pareja. Además, se refleja lo que Tafalla (2001) llama "violencia enmascarada de amor", cuando se le confunde con conductas de preocupación y afecto. Fue posible corroborar también, que existen ciertas idealizaciones en torno a lo que son las relaciones de pareja, así como una tendencia a sobrevalorar los roles tradicionales masculinos de cuido y protección hacia la mujer, por lo que, a veces, en los casos en que esta protección se convierte en control, es desapercibida.

Puede afirmarse, a raíz de estos hallazgos, que no puede desligarse el tema de apego del de género, pues, la forma de vinculación con las y los cuidadores primarios está ligada al contexto social en que se encuentren inmersas las personas, por lo que la construcción del apego estaría influenciada por el rol de género y la socialización, que a partir de él, se da para cada quien, según sea hombre o mujer.

El tiempo que esperaron las participantes para establecerse con una nueva pareja después de finalizar la relación de violencia es otro factor que refleja sus estilos de apego. En el caso de las participantes con estilos de apego ansioso, en el transcurso no mayor del año después de finalizada la relación establecieron un nuevo noviazgo. En el caso de las participantes con tendencias de apego 
temerosas esto no fue así, y, por el contrario, manifestaron grandes deseos de estar solas por mucho tiempo y evitar vinculaciones cercanas, lo cual evidencia su dificultad para la apertura emocional.

Por otra parte, el estudio se enfrentó a varios retos metodológicos pues, no es común que las adolescentes reconozcan y hablen sobre la violencia dentro de sus relaciones de noviazgo, por lo que encontrar posibles casos fue difícil. Además, el tema en cuestión podía generar resistencias en las participantes, por lo que emplear un método principalmente cualitativo fue provechoso para poder llevar a cabo las entrevistas al ritmo de las mujeres. Además se logró profundizar en la vivencia de las participantes, para explorar la dinámica subjetiva de ellas y sus percepciones de la situación de violencia a partir de la experiencia vivida.

Debido a esto, se decide aplicar la historia de vida antes de la EAP y el AEA, para que las participantes no se sintieran inhibidas al hablar de aspectos específicos de sus vidas, sin antes haber hecho referencia a situaciones más generales. Además, el hecho de que fuese una mujer quien realizó las entrevistas ayudó a crear un espacio menos amenazante: de más confianza y empatía con cada una de las participantes.

Se trabajó con un número pequeño de participantes, dadas las características de la investigación, en que no se buscaba generalizar los resultados, sino profundizar en las vivencias individuales de las participantes en relación a su experiencia de violencia en el noviazgo. Por ello, la utilización de tres instrumentos: historia de vida, EAP y AEA, fue provechosa. Al integrarlos por medio de la triangulación, se logró un análisis integral de la situación, evitando estudiar el apego como una variable aislada.

La historia de vida resultó una estrategia fundamental para la recolección de datos en la investigación y permitió que el estudio no se limitara a una simple descripción de estilos de apego. Este instrumento permitió visualizar el apego como una construcción que inicia desde etapas tempranas del desarrollo y facilitó una perspectiva amplia del tema de violencia tomando en consideración experiencias pasadas en la vida de las participante: relaciones intrafamiliares y extrafamiliares, relaciones de violencia y construcción del estilo de apego. De no tomar en cuenta estos factores, la investigación se hubiese centrado en la situación de violencia y el estilo de apego como situaciones aisladas, sin considerar aspectos del contexto y la historia personal, que juegan un papel importante a la hora de abordar esta temática.

Al integrar la historia de vida con la EAP se analiza la construcción del estilo de apego puntuado y las manifestaciones de este a lo largo del discurso de las participantes. En el AEA, no se mostró 
concordancia con estos dos instrumentos y fue gracias a la información obtenida por medio de la historia de vida que se determinó cuál de las dos mediciones de apego resultaba más coherente con el relato de las participantes (en este caso la EAP). Puede considerarse que el AEA, por tratarse de un instrumento de autoinforme y con ítems con claras descripciones acerca de los estilos de apego, puede dar la impresión de que unas opciones son mejores que otras y sesgar las respuestas en términos de "lo que se debería ser".

En síntesis, analizar el tema de la violencia dentro de las relaciones de noviazgo desde la teoría del apego, permitió integrar múltiples variables (roles tradicionales de género, dinámica intrafamiliar, sistema patriarcal y contexto sociocultural), que influyen en la construcción del estilo de apego y en la permanencia en relaciones de violencia por parte de las participantes, siendo este un aporte importante para futuras investigaciones.

\section{Referencias bibliográficas}

Adelman, M. y Kil, S. (2007). Dating conflicts: rethinking dating violence and youth conflict. Violence against women, 13 (12), 1296-1318.

Allen, J. y Manning, N. (2007). From safety to affect regulation: Attachment from the vantage point of adolescence. New directions for child an adolescent development, 117, 23-39.

Ammaniti, M. y Sergi, G. (2003). Clinical dynamics during adolescence: Psychoanalytic and Attachment perspectives. Psychology Department, La Sapienza, University of Rome. Manuscrito o publicación independiente.

Arnett, J. (2000). Emerging Adulthood: A theory of development from the late teens through the twenties. American Psychologist, 55 (5), 469 - 480.

Arnett, J. (2001). Conceptions of the transition to adulthood: Perspectives from adolescence through midlife. Journal of adult development, 8 (2), 133-143.

Arnett, J. (2007). Socialization in emerging adulthood: From the family to the wider world, from socialization to self-socialization. En Grusec y Hastings (Comps.)

Artavia, C. y Carranza, M. (2012). Estilos de apego de mujeres que permanecieron expuestas a situaciones de violencia en sus relaciones de noviazgo durante la adolescencia tardía. Tesis para optar por el grado de Licenciatura en Psicología, Universidad de Costa Rica.

Bartholomew, K. y Horowitz, L. (1991). Attachment styles among young adults: a test of a four -category model. Journal of Personality and Social Psychology, 61(2), 226-244. 
Beck, U. y Beck-Gernsheim, E. (2001). El normal caos del amor. Barcelona, España: Paidós contextos - El Roure.

Berman, S.; Weems, C.; Rodríguez, E.; Zamora, I. (2005). The relation between identity status and romantic attachment style in middle and late adolescence. Journal of adolescence, 29, 737-748.

Bixio, C. (2007). Infancia vs. Minoridad. Centro de investigaciones "María Saleme de Burnichón”, 5(4), 71-83.

Black, B., Tolman, R., Callahan, M., Saunders, D y Weisz, A. (2008). When will adolescents tell someone about dating violence victimization? Violence against women, 14 (7),741758.

Bluck, S. y Habermas, T. (2001). Extending the study of autobiographical memory: thinking back about life across the life span. Review of General Psychology, 5 (2), 135-147.

Bowlby, J. (1982). El apego y la pérdida I: El Apego. Barcelona, España: Paidós.

Bowlby, J. (1998). El apego y la pérdida II: La Separación. Barcelona, España: Paidós.

Bowlby, J. (1983). La pérdida afectiva: Tristeza y depresión. Barcelona, España: Paidós.

Campos, D. (2005). Las orientaciones culturales en relación con el apego hacia los pares y la identidad personal en adolescentes de 15 a 17 años del cantón de Valverde Vega: contribución al estudio de la correspondencia entre la afectividad y la cultura. Tesis para optar por el grado de Licenciatura en Psicología, Universidad de Costa Rica.

Carrillo,S.; Maldonado, C.; Saldarriaga, L.; Vega, L. y Díaz, S. (2004). Patrones de apego en familias de tres generaciones: Abuela, madre adolescente, hijo. Revista Latinoamericana de Psicología.36(3), 409-430.

Castro, R. (2006). Estilos y figuras de apego predominantes en la adolescencia: estimación de la validez y la coherencia interna del "cuestionario de estilos de apego" en el contexto costarricense. Tesis para optar por el grado de Licenciatura en Psicología, Universidad de Costa Rica.

Cisterna, F. (2005) Categorización y triangulación como proceso de validación del conocimiento en investigación cualitativa. Theoria 14 (1), 61-71

Coleman, J. y Hendry, L. (2003). Psicología de la adolescencia. Madrid, España: Ediciones Morata. 
Collins, A., Welsh, D. y Furman, W. (2009). Adolescent romantic relationships. Annual Review of Psychology, 60, 631-652.

Corsi, J.; Auman, V.; Monzon, L. (2003). Maltrato y abuso en el ámbito doméstico: Fundamentos teóricos para el estudio de la violencia en las relaciones familiares. En Jorge Corsi (Comp.). Buenos Aires, Argentina: Paidós.

Delgado, I; Delgado, O. y Sánchez-Queija, I. (2011). Apego a los iguales durante la adolescencia y la adultez emergente. Anales de Psicología, 27(1),155-163.

Feeney, J. y Noller, P. (2001). Apego adulto. Bilbao, España: Biblioteca de Psicología, Desclée de Brouwer.

González, J. (2001). Psicopatología de la adolescencia. México, D.F - Santafé de Bogotá: Manual Moderno.

Grossman, K. y Grossman, K. (2006). Attachment from infancy to adulthood: the major longitudinal studies. New York: The Guilford Press.

Helweg-Larsen, M., Harding, H., y Kleinman, K. (2008). Risk perceptions of dating violence among college women: the role of experience and depressive symptoms. Journal of social and clinical Psychology, 27 (6), 600-620.

Hernández, S., Fernández, C., Baptista, L. (2007) Fundamentos de metodología de la investigación. Madrid, España: McGrawHill 1. ed.

Hidalgo, A. (2009). Femicidio en Costa Rica: 2000-2004. San José, Costa Rica: INAMU; CEFEMINA.

Lafontaine, M.; Lussier, Y. (2005) Does Anger Towards Partner Mediate and Moderate the Link Between Romantic Attachment and Intimate Violence?. Journal of Family Violence, 20 (6), 349-361.

Marquart, B., Nannini, D., Edwards, R., Stanley, L y Wayman, J. (2007). Prevalence of dating violence and victimization: regional and gender differences. Adolescence, 42 (168), 645657.

Mikulincer, M. y Shaver, P. (2007). Attachment in Adulthood: Structure, Dynamics, and Change. New York: The Guilford Press.

Murillo, M. (1996). Reflexión con adolescentes en torno a formas de violencia en sus relaciones de noviazgo. Un estudio exploratorio con adolescentes de 16 a 18 años, de colegios 
públicos del parea metropolitana de San José. Tesis para optar por el grado de Licenciatura en Psicología, Universidad de Costa Rica.

Murillo, M. (1997). Noviazgo entre adolescentes: la puerta de entrada para la vida de pareja adulta. Revista Costarricense de Psicología, (28), 25-38.

Navarro, P.; Díaz, C.(1999). Métodos y técnicas de investigación en Ciencias Sociales. En Delgado, J y Gutiérrez, J (Comps.). Madrid, España: Síntesis Psicología.

Ortiz, M. \& Morales, I. (1999) ¿La Violencia Domestica es Percibida por mujeres de mediana edad?. Revista Cubana de Medicina General Integral, 5 (15), 503-510.

Penagos, A., Rodríguez, M., Carrillo, S y Castro, J. (2006). Apego, relaciones románticas y autoconcepto en adolescentes bogotanos. Univ. Psychol. Bogotá (Colombia), 5 (1), 21-36.

Retana, M (2007). Adolescencia y noviazgo: Formas en que se puede manifestar y reproducir conductas violentas hacia la pareja en las relaciones de noviazgo. Tesis para optar por el grado de Licenciatura en Trabajo Social, Universidad de Costa Rica.

Rey, C. (2008). Prevalencia, factores de riesgo y problemáticas asociadas con la violencia en el noviazgo: una revisión de la literatura. Avances en Psicología Latinoamericana, 26 (2), 227 241.

Sagot, M. y Guzmán, L. (2004). Encuesta nacional de violencia contra las mujeres. Centro de Investigación en Estudios de la Mujer, Universidad de Costa Rica.

Sandoval, C. (2002). Investigación Cualitativa. Programa de especialización en teoría, métodos y técnicas de investigación social. Bogotá, Colombia.

Santamarina, C.; Marinas, J. (1999). Métodos y técnicas de investigación en Ciencias Sociales. En Delgado, J y Gutiérrez, J (Comps.). Madrid, España: Síntesis Psicología.

Silva, F. (s.f). La Violencia Intrafamiliar. Recuperado el 22 de mayo de 2010 de http://www.psicologia-online.com/colaboradores/paola/violencia/index2.shtml

Solano, L. (2007). El abordaje de la prensa escrita sobre el femicidio en Costa Rica en el 2006. Tesis para optar por el grado de Licenciatura en Sociología, Universidad de Costa Rica.

Tafalla, R. (2001). Mujeres, violencia y noviazgo: Fundamentos para un abordaje preventivo desde el trabajo social. Tesis para optar por el grado de Licenciatura en Trabajo Social, Universidad de Costa Rica.

Tapia, J. (en preparación). El desarrollo de la identidad personal: apego y creencias religiosas. Estudio secuencial con jóvenes adolescentes de dos zonas urbanas de San José. Instituto 
de Investigaciones Psicológicas, Vicerrectoría de Investigación $\mathrm{N}^{\circ}$ 723-A3-300, Universidad de Costa Rica.

Yérnoz, Arbiol-Alonso, Plazaola y Sainz (2001) Apego en adultos y percepción de los otros. Anales de Psicología, 17 (002),159-170.

Yérnoz, S. y Comino, P. (2011). Evaluación del apego adulto: Análisis de la convergencia entre diferentes instrumentos. Acción Psicológica, 8(2), 67-85.

Zimmermann, P.; Maier, M.; Winter, M. y Grossman, K. (2001). Attachment and adolescents' emotion regulation during a joint problem-solving task with a friend. International Journal of Behavioral Development. 25(4), 331-343. 